\title{
Robust Circadian Rhythm and Parathyroid Hormone-Induced Resetting during Hypertrophic Differentiation in ATDC5 Chondroprogenitor Cells
}

\author{
Toshihiro Hosokawa ${ }^{1,2,3}$, Yoshiki Tsuchiya ${ }^{1}$, Naoki Okubo ${ }^{1,2,3}$, Tatsuya Kunimoto ${ }^{1,2,3}$, \\ Yoichi Minami ${ }^{1,2}$, Hiroyoshi Fujiwara ${ }^{3}$, Yasuhiro Umemura ${ }^{1}$, Nobuya Koike ${ }^{1}$, \\ Toshikazu Kubo ${ }^{2,3}$ and Kazuhiro Yagita ${ }^{1,2}$
}

${ }^{I}$ Department of Physiology and Systems Bioscience, Kyoto Prefectural University of Medicine, Kawaramachi-Hirokoji, Kamigyo-ku, Kyoto, Japan, ${ }^{2}$ Department of Musculoskeletal Chronobiology, Graduate School of Medical Science, Kyoto Prefectural University of Medicine, Kawaramachi-Hirokoji, Kamigyo-ku, Kyoto, Japan and ${ }^{3}$ Department of Orthopaedics, Graduate School of Medical Science, Kyoto Prefectural University of Medicine, Kawaramachi-Hirokoji, Kamigyo-ku, Kyoto, Japan

Received July 28, 2015; accepted September 4, 2015; published online November 10, 2015

\begin{abstract}
Cartilage tissues possess intrinsic circadian oscillators, which influence chondrocyte function and chondrocyte specific gene expression. However, it is not fully understood how chondrogenesis influences the circadian clock, and vice versa. Thus, we established ATDC5 cells which were stably transfected with the Bmal1:luc reporter and revealed robust circadian rhythms in ATDC5 cells during differentiation. Moreover, the circadian clock in ATDC5 cells was strongly reset by PTH in a circadian time-dependent manner. This assay system is expected to be useful for investigating the role of the circadian clock in chondrogenic differentiation and the precise molecular mechanisms underlying PTH action on the chondrocyte circadian clock.
\end{abstract}

Key words: circadian rhythm, chondrogenic differentiation, ATDC5, parathyroid hormone

\section{Introduction}

The circadian clock controls diverse behavioral and physiological processes including activity, feeding, sleep/ wake cycles, and energy metabolisms $[1,18]$. The master pacemaker resides in the suprachiasmatic nucleus (SCN) of the hypothalamus and coordinates peripheral oscillators in multiple organ and tissues throughout the body [26]. The intracellular circadian molecular oscillator is thought to be generated by the transcriptional and translational feedback loop comprised of a set of clock genes such as Bmall, Clock, Per1, Per2, Cry1, Cry2, Rev-erb $\alpha$, Rev-erb $\beta$, and Rora [15]. The transcription factors BMAL1 and CLOCK heterodimerize and bind to E-box sequences at the promoter regions of Per and Cry to promote their transcription.

Correspondence to: Kazuhiro Yagita, Department of Physiology and Systems Bioscience, Kyoto Prefectural University of Medicine, Kawaramachi-Hirokoji, Kamigyo-ku, Kyoto 602-8566, Japan. E-mail: kyagita@koto.kpu-m.ac.jp
PER and CRY proteins translocate into nuclei and inhibit their own transcription. REV-ERB $\alpha$, REV-ERB $\beta$, and ROR $\alpha$ regulate the circadian expression of Bmall via the retinoid-related orphan receptor response element (RORE). These transcriptional loops enable circadian oscillation of clock and clock-controlled gene expression.

A number of studies have demonstrated that bones have intrinsic circadian clocks and that bone turnover, mineralization, and metabolism all follow circadian rhythms [4, $8,19,28,34]$. It has also been shown that the deletion of core clock genes such as Per1, Per2, Cryl, Cry2, and Bmall affect bone metabolism [5, 17]. Several lines of evidence indicate that circadian oscillators also reside in growth plates and articular cartilage $[4,7,23,29]$. The importance of the circadian clock component in chondrocytes has been suggested by a previous study which demonstrated that chondrocyte-specific Bmall deficiency causes reduced bone length and decreased expression of $I h h$, which is a major regulator of chondrocyte hypertrophy [29]. As chondrocyte differentiation is a key process during 
endochondral ossification within developing and growing bones as well as during fracture healing, it is important to delineate the characteristics and functions of the circadian clock in chondrocytes during differentiation. However, it is not fully understood whether and how chondrocyte differentiation influences the circadian clock, and vice versa. We therefore aimed to establish an assay system that would enable real-time monitoring of the circadian clock during stage-controlled chondrogenic differentiation.

In this study, the establishment of a system for the real-time monitoring of the intracellular circadian clockwork in the chondrogenic cell line ATDC5 is reported on. ATDC5 cells, originally derived from mouse teratocarcinomas, have been widely used in many studies as a model for chondrocyte differentiation [3, 22, 27]. Importantly, it has been reported that ATDC5 cells exhibit prechondrocytic characteristics and recapitulate on the overall in vivo hypertrophic differentiation, mineralization, and calcification of chondrocytes [27]. We have successfully established ATDC5 cells stably transfected with the Bmall promoterdriven luciferase reporter (Bmal1:luc) in order to monitor circadian rhythms in ATDC5 during chondrogenic differentiation. Furthermore, we investigated the effects of parathyroid hormones (PTH) on the circadian clock in differentiated ATDC5 cells to clarify PTH action on the chondrocyte circadian clock.

\section{Materials and Methods}

\section{Cell cultures}

ATDC5 cells were obtained from the RIKEN cell bank (Tsukuba, Japan). ATDC5 cells were cultured in a 1:1 mixture of Dulbecco's modified Eagle's medium and Ham's F-12 medium (DMEM/F12) (Wako) containing 5\% FBS, 100 units $/ \mathrm{ml}$ penicillin, and $100 \mu \mathrm{g} / \mathrm{ml}$ streptomycin (Nacalai Tesque) at $37^{\circ} \mathrm{C}$ under $5 \% \mathrm{CO}_{2}$. The medium was changed every two or three days.

\section{Differentiation of ATDC5 cells}

After the cells reached confluence, the medium was changed to a differentiating medium that contained DMEM/F12, 5\% FBS, 100 units $/ \mathrm{ml}$ penicillin, $100 \mu \mathrm{g} / \mathrm{ml}$ streptomycin, $10 \mu \mathrm{g} / \mathrm{ml}$ bovine insulin (SIGMAALDRICH), $10 \mu \mathrm{g} / \mathrm{ml}$ human transferrin (Calbiochem), and $3 \times 10^{-8} \mathrm{M}$ sodium selenite (SIGMA-ALDRICH). The medium was changed every two or three days.

\section{Establishment of ATDC5 cells stably transfected with the Bmall:luc reporter}

To establish ATDC5 cells stably transfected with the Bmal1:luc reporter, ATDC5 cells were plated in a $35-\mathrm{mm}$ dish at a density of $1.0 \times 10^{5}$ cells/dish and cultured overnight. For transfection, $6 \mu \mathrm{l}$ of FuGENE6 transfection Reagent (Promega) was diluted in $94 \mu \mathrm{l}$ of DMEM/F12 and incubated for $5 \mathrm{~min}$ at room temperature. Then, $1 \mu \mathrm{g}$ of Bmal1:luc-pT2A plasmid with a Zeocin selection marker and $1 \mu \mathrm{g}$ of Tol 2 transposase expression vector (pCAGGSTP) [32] were mixed with FuGENE6 transfection Reagent medium and incubated for $15 \mathrm{~min}$ at room temperature. The mixture was added to the culture medium of ATDC5 cells seeded in a $35-\mathrm{mm}$ dish. Four hours after transfection, the medium was changed to a fresh medium. The stably transfected cells were selected with $20 \mu \mathrm{g} / \mathrm{ml}$ of Zeocin (Invitrogen) for 12 days.

\section{RNA extraction and quantitative real-time PCR}

Total RNA was isolated from the ATDC5 cells using acid guanidinium thiocyanate-phenol-chloroform extraction with Isogen (Nippon Gene). For cDNA synthesis, $1 \mu \mathrm{g}$ of the total RNA was reverse-transcribed using M-MLV Reverse Transcriptase (Invitrogen) and a random primer (Takara). Real-time PCR was performed using iTaq Universal SYBR Green Supermix (Biorad) and a StepOnePlus real-time PCR system (Applied Biosystems). mRNA expression levels were normalized to $18 \mathrm{~S}$ rRNA. Primer sequences were as follows: Collagen type II (Col2a1) forward primer 5'-CGGTGTCAAGGGTCACAGAG-3', Col2al reverse primer 5'-ACCAGGGGAACCACTCTCA C-3', Aggrecan forward primer 5'-CTGCTTCGCTGAGGA GATG-3', Aggrecan reverse primer 5'-AGGTAGAGCTGG CCTGTGG-3', Collagen typeX (Col10a1) forward primer 5'-GTACCAAACGCCCACAGG-3', Col10a1 reverse primer 5'-CTGGTGGACCAGGAATGC-3', 18S rRNA forward primer 5'-CGCCGCTAGAGGTGAAATTC-3', 18S rRNA reverse primer 5'-CGAACCTCCGACTTTCGTTCT$3^{\prime}$.

\section{Alcian blue staining}

On day 0 and day 21 of the differentiation culture, ATDC5 cells were rinsed with PBS three times and fixed with $95 \%$ ethanol for $20 \mathrm{~min}$. Then, the cells were stained with alcian blue 8GS (SIGMA-ALDRICH) in $0.1 \mathrm{M} \mathrm{HCl}$ for $2 \mathrm{hr}$ at room temperature and rinsed with distilled water.

\section{Real-time bioluminescence recording}

ATDC5 cells were cultured in 24-well black plates. For bioluminescence recording, the medium was changed with a monitoring medium, which contained phenol-redfree DMEM/Ham's F-12 (Nacalai Tescque), 5\% FBS, 100 units/ml penicillin, $100 \mu \mathrm{g} / \mathrm{ml}$ streptomycin (Nacalai Tesque), $0.2 \mathrm{mM}$ luciferin (Promega), and $100 \mathrm{nM}$ dexamethasone. The plates were placed in photomultiplier tube-based real-time bioluminescence monitoring equipment kept at $35^{\circ} \mathrm{C}$. Bioluminescence was measured and integrated for one min in $20 \mathrm{~min}$ intervals.

\section{PTH treatment}

Human PTH (1-34) was purchased from Peptide Institute, Inc. (Ibaraki, Japan) and was dissolved in distilled water. Forty-one hours or $50 \mathrm{hr}$ after synchronization, PTH (1-34) was added to the medium at a dose of $10^{-10} \mathrm{M}, 10^{-9}$ $\mathrm{M}$, or $10^{-8} \mathrm{M}$. For acquiring a phase response curve, $10^{-8} \mathrm{M}$ 
PTH (1-34) was added to the medium at the indicated time after synchronization.

\section{Analysis of bioluminescence data}

The raw data were detrended through the subtraction of the $24 \mathrm{hr}$ moving average from the raw data. To calculate the phase of the rhythms, the detrended data from $86 \mathrm{hr}$ to $162 \mathrm{hr}$ after synchronization were fitted to a sine wave using the least-square method. We used the following equation:

$$
y(t)=A e^{-k t} \sin \left(\frac{2 \pi(t-\varphi)}{\tau}\right)
$$

where $A=$ amplitude, $k=$ damping constant, $t=$ time, $\tau=$ period, and $\varphi=$ phase.

\section{Data analysis}

Two group comparisons were performed using Students' $t$ tests. The Bonferroni correction was applied for multiple comparisons.

\section{Results}

\section{Establishment of ATDC5 cells stably transfected with the Bmal1 promoter-driven luciferase reporters}

To monitor the internal circadian rhythms in ATDC5 cells, we introduced a Bmall promoter-driven luciferase reporter into the cells using the Tol2 transposon-based gene transfer system [32]. The established ATDC5 $5^{\text {Bmall:luc }}$ cells should express the luciferase reporter gene under the control of the Bmall promoter. To investigate the nature of the intrinsic circadian clock during chondrogenic differentiation of ATDC5 cells, ATDC5 $5^{\text {Bmall:luc }}$ cells were differentiated and Bmal1:luc bioluminescence was measured upon dexamethasone treatment of the cells. As a result, the robust circadian oscillation of Bmall:luc bioluminescence was observed from 0 to 21 days after inducing chondrogenic differentiation (Fig. 1A). The expression levels of chondrocyte-differentiation markers such as Col2a1, Aggrecan, and Col10a1 were significantly increased during chondrogenic differentiation of ATDC5 $5^{\text {Bmall:luc }}$ cells (Fig. 1B). In addition, alcian blue staining clearly demonstrated that the matrix proteoglycan was synthesized 21 days after inducing the chondrogenic differentiation of the ATDC5 $5^{\text {Bmall:luc }}$ cells (Fig. 1C). These data collectively indicate that ATDC5 cells keep the intrinsic circadian molecular clock ticking both before and during chondrogenic differentiation

\section{PTH induces phase shifts of circadian rhythms in ATDC5 cells}

We have previously shown that PTH resets circadian rhythms in an ex vivo culture of femurs [24]. Although PTH affected the rhythms observed in growth plate cartilage and articular catilage, the in-depth effect of PTH on the rhythms of chondrocytes and/or chondrogenic cells has remained unclear. Thus, we specifically examined the effects of PTH on circadian rhythms in ATDC5 $5^{\text {Bmall:luc }}$ cells. The cells were treated with dexamethasone to synchronize their circadian rhythms after 7 days with the differentiation culture. When the cells were treated with $10^{-10} \mathrm{M}$ of PTH $41 \mathrm{hr}$ after synchronization, Bmall:luc bioluminescence rhythms were not significantly altered (Fig. 2A, B). However, $10^{-9}$ and $10^{-8} \mathrm{M}$ of PTH caused significant phase delays of $4.28(\mathrm{SD} \pm 0.65) \mathrm{hr}$ and $7.79(\mathrm{SD} \pm 0.44) \mathrm{hr}$, respectively (Fig. 2A, B). Administration of $10^{-9}$ and $10^{-8} \mathrm{M}$ of PTH $50 \mathrm{hr}$ after synchronization resulted in significant phase advances of $3.99(\mathrm{SD} \pm 0.16) \mathrm{hr}$ and $2.85(\mathrm{SD} \pm 0.18)$ hr, respectively, while $10^{-10} \mathrm{M}$ of PTH had no observable effect on bioluminescence rhythms (Fig. 2A, B). These results indicate that $\mathrm{PTH}$ has the ability to phase-shift circadian rhythms in ATDC5 cells. To address the detailed properties of the PTH-induced resetting of the circadian clock, we examined the phase-dependent effect of PTH throughout the circadian cycle. As a result, it was found that PTH induced a strong phase shift of about $12 \mathrm{hr}$ when administered around the peak time of bioluminescence ( $44 \mathrm{hr}$ after synchronization) (Fig. 2C). On the other hand, PTH did not cause any phase shift when administered around the trough time of bioluminescence ( $56 \mathrm{hr}$ after synchronization) (Fig. 2C). The resultant phase response curve clearly demonstrates that PTH causes strong type 0 phase resetting, which indicates the strong phase shift by up to $12 \mathrm{hr}$ to a common new phase regardless of the initial circadian phases [30]. These results further define PTH as a strong resetting factor of circadian rhythms in chondrocytes.

\section{Discussion}

Cell differentiation is a critical process for cells to acquire specific characteristics and functions over the course of the development, growth, and turnover of diverse types of tissues. The circadian clock has been associated with cell differentiation in various contexts; embryonic stem cell differentiation [31], T cell differentiation [33], epidermal stem cell function [11], regenerative hair cycling [25], and maintenance of skeletal muscle [2]. According to recent studies, circadian regulation of chondrocyte differentiation and function has also been proposed [14, 29]. During endochondral ossification, chondrocytes undergo multistep differentiation processes including prechondrogenic condensation and hypertrophic differentiation, and are ultimately replaced by bone $[16,21]$. However, the properties of the circadian clock in chondrocytes at each differentiation stage remain mostly unclear. In this study, we describe for the first time the circadian clockwork in a synchronized differentiation culture of chondrocytes by utilizing ATDC5 cells and a real-time circadian rhythm monitoring method. ATDC5 cells show characteristics of undifferentiated prechondrogenic cells, have the potential for chondrogenic differentiation, and thus are considered suitable in vitro models for investigating chondrogenesis 
A

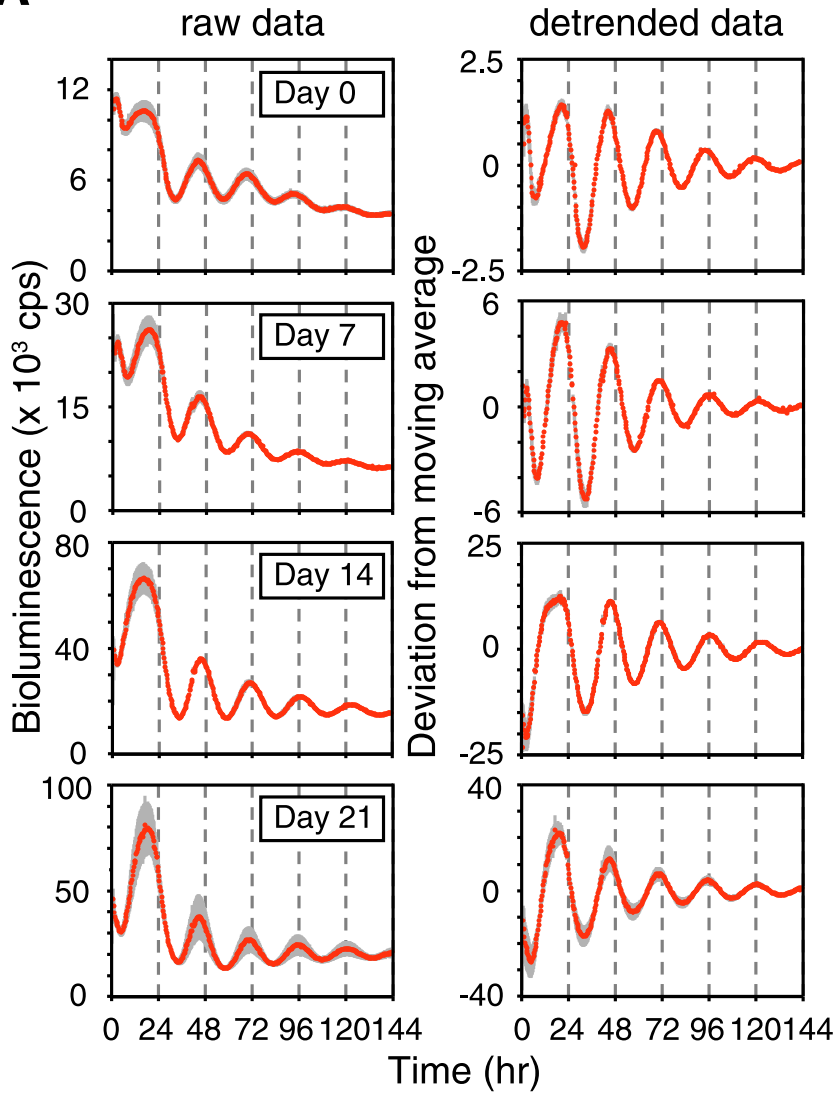

B

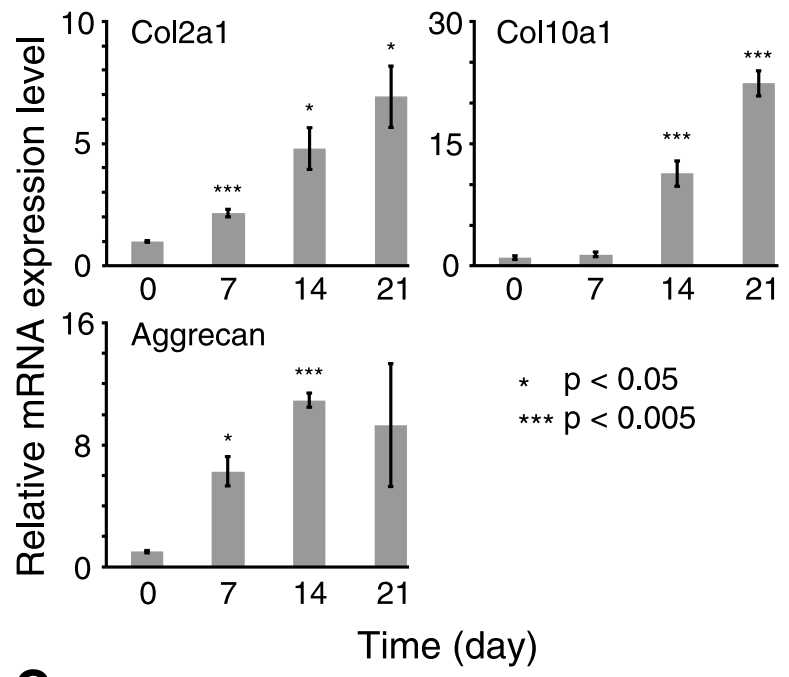

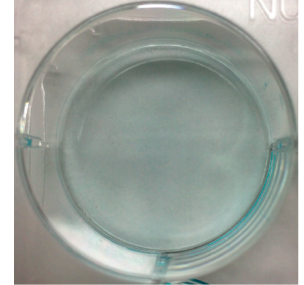

Day 0

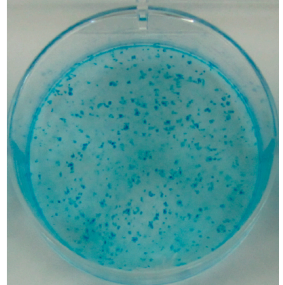

Day 21

Fig. 1. ATDC5 chondroprogenitor cells show robust circadian rhythms during chondrogenic differentiation. A: Bioluminescence traces of ATDC5 $5^{\text {Bmall:luc }}$ cells on differentiation days $0,7,14$, and 21 . Left and right panels show raw and detrended data, respectively. Data shown are means \pm SD ( $\mathrm{n}=3$ per group). B: The relative mRNA expression levels of Col2a1, Aggrecan, and Col10a1 in ATDC5 $5^{\text {Bmall:luc }}$ cells on differentiation days 0, 7, 14, and 21. Data shown are means \pm SD ( $=3$ per group; *, $\mathrm{p}<0.05$; $^{* *}, \mathrm{p}<0.01 ; * * *, \mathrm{p}<0.005$ (vs. day 0$)$ ). C: Alcian blue staining of ATDC5 ${ }^{\text {Bmall:luc }}$ cells on day 0 and 21 .

[27]. Intriguingly, although the induction of chondrogenic differentiation triggers drastic changes in cell morphology, anabolic activity, and gene expression profiles in ATDC5 cells, the circadian clockwork seems to be equivalently ticking. This suggests robustness of the chondrocyte clock over the course of chondrogenic differentiation.

The fact that the PTH resets circadian rhythms in differentiating ATDC5 cells underscores the function of PTH in the regulation of the chondrocyte circadian clock. In growth plate cartilage, a PTH-related peptide (PTHrP) that is secreted by perichondrial cells and chondrocytes functions to keep chondrocytes in their proliferative stage and inhibit their hypertrophic differentiation. At the same time, chondrocytes in the prehypertrophic and hypertrophic zones secrete Ihh, which promotes PTHrP production at the ends of bones, and thus prevents further hypertrophic differentiation, exerting a negative feedback type of regulation [13]. Given that the circadian clock could induce Ihh expression rhythms [29], it is possible that production of Ihh and PTHrP may be regulated in a circadian manner, and in turn the Ihh-PTHrP axis may influence the circadian clock in a paracrine manner to synchronize the chondrocyte clock within the growth plate. The underlying mechanisms of the PTH-induced phase shift are unclear. PTH and PTHrP bind to the common receptor PTH1R, leading to the activations of both the cAMP/protein kinase A (PKA) pathway and the phospholipase $\mathrm{C} /$ protein kinase $\mathrm{C}$ (PKC) pathway [6]. As PTH has been reported to induce the transient expression of Per1 mRNA in ATDC5 cells via PKA-cAMP response element-binding protein (CREB) signaling [9], PTH-mediated resetting of the circadian clock in ATDC5 cells might be dependent on PKA activity. Future studies will be needed to elucidate intracellular PTH1R signaling that leads to the resetting of circadian rhythms in chondrocytes.

Intermittent administration of PTH-analog teriparatide (recombinant human PTH (1-34)) is widely used for the treatment of osteoporosis, because it accelerates the bone remodeling rate [10]. In fracture healing, teriparatide has been shown to enhance endochondral bone repair by facilitating the recruitment and differentiation of chondrocytes [12]. Interestingly, the timing of teriparatide administration may influence its efficacy for the treatment of postmenopausal osteoporosis [20]. As the circadian clock regulates many aspects of behavior and physiology and diverse functions of peripheral tissues including cartilages, it is clini- 
A

\section{$41 \mathrm{hr}$}
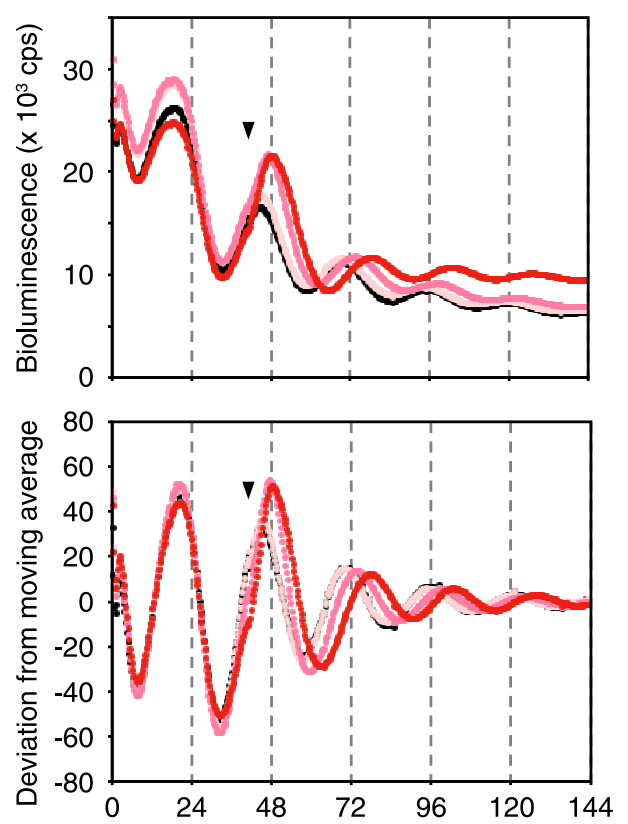

Time (hr)
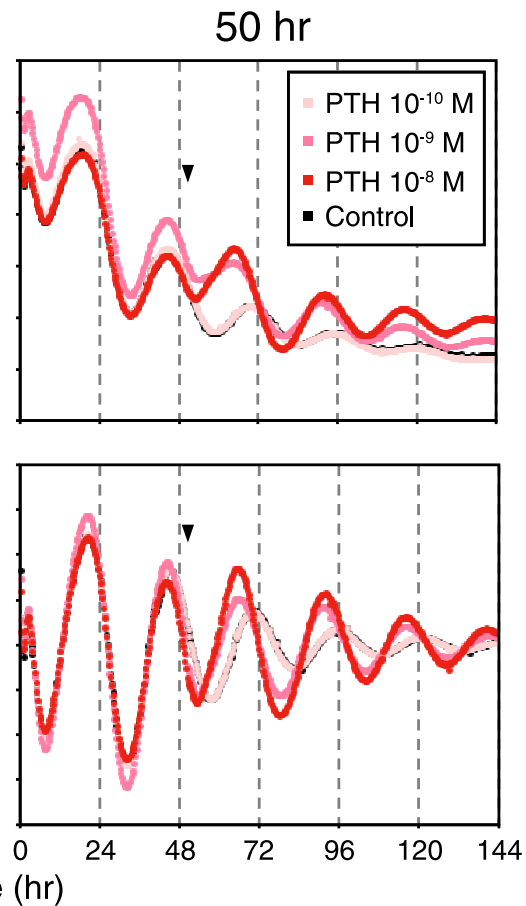

B

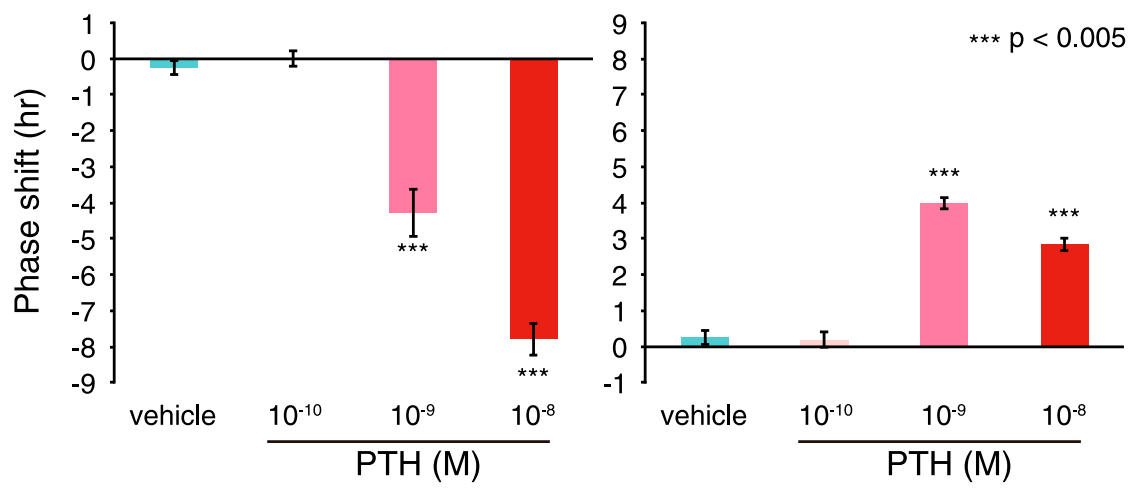

C

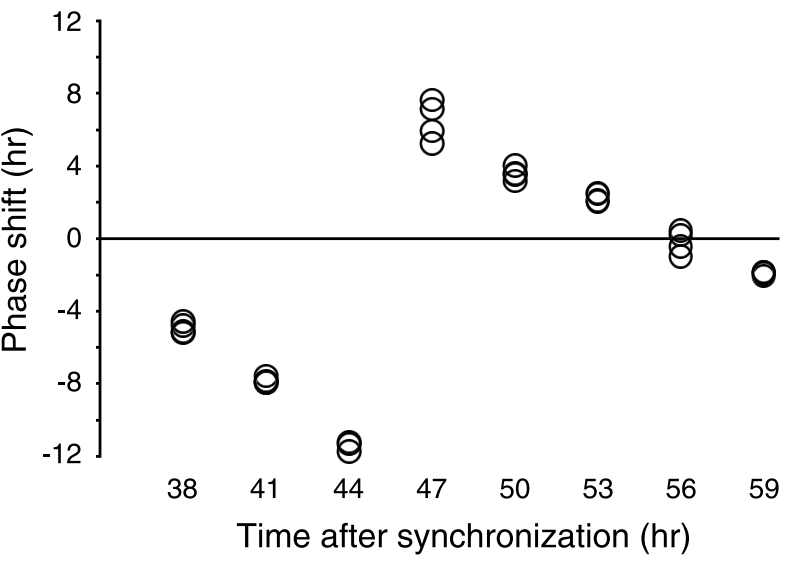

Fig. 2. PTH (1-34) induces a strong phase shift of circadian rhythms in ATDC5 cells. A: The phase shift of circadian bioluminescence rhythms by PTH

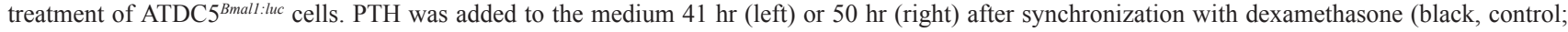
pale pink, $10^{-10} \mathrm{M} \mathrm{PTH}$; pink, $10^{-9} \mathrm{M} \mathrm{PTH}$; red, $\left.10^{-8} \mathrm{M} \mathrm{PTH}\right)$. The upper and lower panels show the raw and detrended data, respectively. B: Quantitative analysis of the PTH-induced phase shift. The indicated concentrations of PTH and vehicle were administered $41 \mathrm{hr}$ (left) and $50 \mathrm{hr}$ (right) after synchronization. The value of the phase shift was determined by sine wave fitting ( $\mathrm{n}=4$ per group; $* * *, p<0.005$ (vs. vehicle)). C: The phase response curve shows phase shifts by $10^{-8} \mathrm{M}$ PTH treatment at indicated time points. Each closed circle indicates the value calculated from one trace of bioluminescence rhythms. 
cally important to know the optimal timing for teriparatide administration so that the internal circadian clock will not be perturbed. In addition, it will also be meaningful to clarify the functional significance of the PTH-induced resetting of the chondrocyte circadian clock. In this study, we demonstrate that ATDC5 cells show clear and robust circadian rhythms throughout the differentiation process from chondroprogenitor cells to hypertrophic chondrocytes. It is expected that the ATDC5 $5^{\text {Bmall:luc }}$ cells will provide a useful assay system to assess the circadian time-dependent properties of chondrocyte function and differentiation, where PTH regulates both chondrogenesis and the circadian clock.

\section{Acknowledgments}

We give special thanks to all member of Yagita Lab for discussion. We also thank Dr. H Kim for helping the ATDC5 culture for circadian rhythm analysis. Grants-inAid for Scientific Research were from the Japan Society for the Promotion of Science (JSPS) (to Y. U., Y. M., and K. Y.).

\section{Conflict of Interest}

The authors declare no competing financial interest.

\section{References}

1. Albrecht, U. (2012) Timing to perfection: the biology of central and peripheral circadian clocks. Neuron 74; 246-260.

2. Andrews, J. L., Zhang, X., McCarthy, J. J., McDearmon, E. L., Hornberger, T. A., Russell, B., Campbell, K. S., Arbogast, S., Reid, M. B., Walker, J. R., Hogenesch, J. B., Takahashi, J. S. and Esser, K. A. (2010) CLOCK and BMAL1 regulate MyoD and are necessary for maintenance of skeletal muscle phenotype and function. Proc. Natl. Acad. Sci. U S A 107; 19090-19095.

3. Atsumi, T., Miwa, Y., Kimata, K. and Ikawa, Y. (1990) A chondrogenic cell line derived from a differentiating culture of AT805 teratocarcinoma cells. Cell Differ. Dev. 30; 109-116.

4. Dudek, M. and Meng, Q. J. (2014) Running on time: the role of circadian clocks in the musculoskeletal system. Biochem. J. 463; $1-8$.

5. Fu, L., Patel, M. S., Bradley, A., Wagner, E. F. and Karsenty, G. (2005) The molecular clock mediates leptin-regulated bone formation. Cell 122; 803-815.

6. Gensure, R. C., Gardella, T. J. and Juppner, H. (2005) Parathyroid hormone and parathyroid hormone-related peptide, and their receptors. Biochem. Biophys. Res. Commun. 328; 666678 .

7. Gossan, N., Zeef, L., Hensman, J., Hughes, A., Bateman, J. F., Rowley, L., Little, C. B., Piggins, H. D., Rattray, M., BootHandford, R. P. and Meng, Q. J. (2013) The circadian clock in murine chondrocytes regulates genes controlling key aspects of cartilage homeostasis. Arthritis Rheum. 65; 2334-2345.

8. Greenspan, S. L., Dresner-Pollak, R., Parker, R. A., London, D. and Ferguson, L. (1997) Diurnal variation of bone mineral turnover in elderly men and women. Calcif. Tissue Int. 60; 419423.

9. Hinoi, E., Ueshima, T., Hojo, H., Iemata, M., Takarada, T. and Yoneda, Y. (2006) Up-regulation of per mRNA expression by parathyroid hormone through a protein kinase A-CREB- dependent mechanism in chondrocytes. J. Biol. Chem. 281; 23632-23642

10. Hodsman, A. B., Bauer, D. C., Dempster, D. W., Dian, L., Hanley, D. A., Harris, S. T., Kendler, D. L., McClung, M. R., Miller, P. D., Olszynski, W. P., Orwoll, E. and Yuen, C. K. (2005) Parathyroid hormone and teriparatide for the treatment of osteoporosis: a review of the evidence and suggested guidelines for its use. Endocr. Rev. 26; 688-703.

11. Janich, P., Toufighi, K., Solanas, G., Luis, N. M., Minkwitz, S., Serrano, L., Lehner, B. and Benitah, S. A. (2013) Human epidermal stem cell function is regulated by circadian oscillations. Cell Stem Cell 13; 745-753.

12. Kakar, S., Einhorn, T. A., Vora, S., Miara, L. J., Hon, G., Wigner, N. A., Toben, D., Jacobsen, K. A., Al-Sebaei, M. O., Song, M., Trackman, P. C., Morgan, E. F., Gerstenfeld, L. C. and Barnes, G. L. (2007) Enhanced chondrogenesis and Wnt signaling in PTH-treated fractures. J. Bone Miner. Res. 22; 1903-1912.

13. Kronenberg, H. M. (2003) Developmental regulation of the growth plate. Nature 423; 332-336.

14. Le, N. Q., Binh, N. T., Takarada, T., Takarada-Iemata, M., Hinoi, E. and Yoneda, Y. (2013) A negative correlation between Per1 and Sox6 expression during chondrogenic differentiation in prechondrocytic ATDC5 cells. J. Pharmacol. Sci. 122; 318-325.

15. Lowrey, P. L. and Takahashi, J. S. (2011) Genetics of circadian rhythms in Mammalian model organisms. Adv. Genet. 74; 175230.

16. Mabvuure, N., Hindocha, S., Jordan, D. and Khan, W. S. (2012) Chondrogenesis and developments in our understanding. Curr. Stem Cell Res. Ther. 7; 243-259.

17. Maronde, E., Schilling, A. F., Seitz, S., Schinke, T., Schmutz, I., van der Horst, G., Amling, M. and Albrecht, U. (2010) The clock genes Period 2 and Cryptochrome 2 differentially balance bone formation. PLoS One 5; e11527.

18. Masri, S. and Sassone-Corsi, P. (2013) The circadian clock: a framework linking metabolism, epigenetics and neuronal function. Nat. Rev. Neurosci. 14; 69-75.

19. McElderry, J. D., Zhao, G., Khmaladze, A., Wilson, C. G., Franceschi, R. T. and Morris, M. D. (2013) Tracking circadian rhythms of bone mineral deposition in murine calvarial organ cultures. J. Bone Miner. Res. 28; 1846-1854.

20. Michalska, D., Luchavova, M., Zikan, V., Raska, I. Jr., Kubena, A. A. and Stepan, J. J. (2012) Effects of morning vs. evening teriparatide injection on bone mineral density and bone turnover markers in postmenopausal osteoporosis. Osteoporos. Int. 23; 2885-2891.

21. Michigami, T. (2014) Current understanding on the molecular basis of chondrogenesis. Clin. Pediatr. Endocrinol. 23; 1-8.

22. Newton, P. T., Staines, K. A., Spevak, L., Boskey, A. L., Teixeira, C. C., Macrae, V. E., Canfield, A. E. and Farquharson, C. (2012) Chondrogenic ATDC5 cells: an optimised model for rapid and physiological matrix mineralisation. Int. J. Mol. Med. 30; 1187-1193.

23. Okubo, N., Minami, Y., Fujiwara, H., Umemura, Y., Tsuchiya, Y., Shirai, T., Oda, R., Inokawa, H., Kubo, T. and Yagita, K. (2013) Prolonged bioluminescence monitoring in mouse ex vivo bone culture revealed persistent circadian rhythms in articular cartilages and growth plates. PLoS One 8; e78306.

24. Okubo, N., Fujiwara, H., Minami, Y., Kunimoto, T., Hosokawa, T., Umemura, Y., Inokawa, H., Asada, M., Oda, R., Kubo, T. and Yagita, K. (2015) Parathyroid hormone resets the cartilage circadian clock of the organ-cultured murine femur. Acta Orthop. 86; 627-631.

25. Plikus, M. V., Vollmers, C., de la Cruz, D., Chaix, A., Ramos, R., Panda, S. and Chuong, C. M. (2013) Local circadian clock gates cell cycle progression of transient amplifying cells during regenerative hair cycling. Proc. Natl. Acad. Sci. U S A 110; 
E2106-2115.

26. Reppert, S. M. and Weaver, D. R. (2002) Coordination of circadian timing in mammals. Nature 418; 935-941.

27. Shukunami, C., Shigeno, C., Atsumi, T., Ishizeki, K., Suzuki, F. and Hiraki, Y. (1996) Chondrogenic differentiation of clonal mouse embryonic cell line ATDC5 in vitro: differentiationdependent gene expression of parathyroid hormone (PTH)/PTHrelated peptide receptor. J. Cell Biol. 133; 457-468.

28. Srivastava, A. K., Bhattacharyya, S., Li, X., Mohan, S. and Baylink, D. J. (2001) Circadian and longitudinal variation of serum C-telopeptide, osteocalcin, and skeletal alkaline phosphatase in $\mathrm{C} 3 \mathrm{H} / \mathrm{HeJ}$ mice. Bone 29; 361-367.

29. Takarada, T., Kodama, A., Hotta, S., Mieda, M., Shimba, S., Hinoi, E. and Yoneda, Y. (2012) Clock genes influence gene expression in growth plate and endochondral ossification in mice. J. Biol. Chem. 287; 36081-36095.

30. Winfree, A. T. (1970) Integrated view of resetting a circadian clock. J. Theor. Biol. 28; 327-374.

31. Yagita, K., Horie, K., Koinuma, S., Nakamura, W., Yamanaka, I., Urasaki, A., Shigeyoshi, Y., Kawakami, K., Shimada, S., Takeda,
J. and Uchiyama, Y. (2010) Development of the circadian oscillator during differentiation of mouse embryonic stem cells in vitro. Proc. Natl. Acad. Sci. U S A 107; 3846-3851.

32. Yagita, K., Yamanaka, I., Emoto, N., Kawakami, K. and Shimada, S. (2010) Real-time monitoring of circadian clock oscillations in primary cultures of mammalian cells using Tol2 transposon-mediated gene transfer strategy. BMC Biotechnol. 10; 3.

33. Yu, X., Rollins, D., Ruhn, K. A., Stubblefield, J. J., Green, C. B., Kashiwada, M., Rothman, P. B., Takahashi, J. S. and Hooper, L. V. (2013) TH17 cell differentiation is regulated by the circadian clock. Science 342; 727-730.

34. Zvonic, S., Ptitsyn, A. A., Kilroy, G., Wu, X., Conrad, S. A., Scott, L. K., Guilak, F., Pelled, G., Gazit, D. and Gimble, J. M. (2007) Circadian oscillation of gene expression in murine calvarial bone. J. Bone Miner. Res. 22; 357-365.

This is an open access article distributed under the Creative Commons Attribution License, which permits unrestricted use, distribution, and reproduction in any medium, provided the original work is properly cited. 